\title{
Quenched Magneto-association of Ultracold Feshbach Molecules
}

\author{
Kirk Waiblinger, ${ }^{1}$ Jason R. Williams, ${ }^{2}$ and José P. D'Incao ${ }^{1,3}$ \\ ${ }^{1}$ Department of Physics, University of Colorado, Boulder, Colorado, USA \\ ${ }^{2}$ Jet Propulsion Laboratory, California Institute of Technology, Pasadena, California, USA \\ ${ }^{3}$ JILA, University of Colorado and NIST, Boulder, Colorado, USA
}

\begin{abstract}
We study enhanced magneto-association of atoms into weakly-bound molecules near a Feshbach resonance using a quench preparatory stage. In anticipation of experiments with NASA's Cold Atom Laboratory aboard the International Space Station, we assume as a baseline a dual-species $\left({ }^{87} \mathrm{Rb}\right.$ and ${ }^{41} \mathrm{~K}$ ) gas in a parameter regime enabled by a microgravity environment. This includes subnanokelvin temperatures and dual-species gases at densities as low as $10^{8} / \mathrm{cm}^{3}$. Our studies indicate that, in such a regime, traditional magneto-association schemes are inefficient due to the weak coupling between atomic and molecular states at low-densities, thus requiring extremely long magnetic field sweeps. To address this issue we propose a modified scheme where atoms are quenched to unitarity before proceeding with magneto-association. This substantially improves molecular formation, allowing for up to $80 \%$ efficiency, and within time-scales much shorter than those associated to atomic and molecular losses. We show that this scheme also applies at higher densities, therefore proving to be of interest to ground-based experiments as well.
\end{abstract}

\section{INTRODUCTION}

Ultracold molecules are today one of the physical systems most used to study a variety of physical phenomena, ranging from quantum information [1 5], to ultracold chemistry [6] 9 , to exploration of novel dipolar phases of matter [10 15], to tests of variations of fundamental constants [16 18]. As a result, developing efficient techniques to produce such molecules is a highly sought after goal [19 25]. Since most experiments using such molecules start from a gas of ultracold atoms, the central question is how to efficiently produce a dense sample of molecules while still keeping them at ultracold temperatures. Magneto-association provides such a path and has been routinely implemented to a broad range of molecular experiments to date [26 31]. In this scheme, atoms are exposed to a magnetic field, $B$, tuned near a Feshbach resonance, causing the $s$-wave scattering length, $a$, to go through a pole, and causing interactions to become extremely strong [32]. Sweeping the magnitude of the $B$ field across the resonance will convert atoms adiabatically to weakly bound Feshbach molecules, existing for $a>0$. Feshbach molecules can then be further used to explore a variety of phenomena or can be used as an intermediate state to form more deeply bound molecular species by using other association schemes like STIRAP [33].

The efficiency of magneto-association is fundamentally controlled by the $B$-field sweeping rates, but also depends on the initial atomic densities and temperatures 26 31], as well as depending non-trivially on the microscopic details characterizing the interatomic interactions [19, 20]. For NASA's Cold Atom Laboratory $(\mathrm{CAL})$, a multi-user facility aboard the International Space Station [34, 35], the microgravity environment will provide experimental conditions vastly different than those achievable in ground-based experiments exploring magneto-association. Here, we will show that although the unique experimental conditions available at CAL favor high phase-space density, long interrogation times, and suppression of gravitation-sag, the ultralow atomic densities $\left(n=10^{8}-10^{11} / \mathrm{cm}^{3}\right)$ desired for various proposed experiments will drastically affect the efficiency of magneto-association. At such low densities, as opposed to those typically found in ground-based experiments ( $n=10^{12} / \mathrm{cm}^{3}$ and higher), the required $B$-field sweeps to obtain a satisfactory efficiency are simply too slow, compromising the stability of the molecular sample against three-body losses. A similar scenario is also found near narrow Feshbach resonances where atomic and molecular states are naturally weak. This issue is of particular importance for studies of dual-species atom interferometry where the formation heteronuclear Feshbach molecules 24] is of fundamental interest to mitigate some of the major sources of systematic errors for highprecision tests of fundamental physics 36 38].

In order to optimize the formation of Feshbach molecules, we modify the traditional magneto-association (tMA) scheme. Our scheme adds a preparatory stage where the $B$-field is changed abruptly (quenched) from off-resonance to on-resonance, and then is allowed to dwell in this regime while developing correlations. This strongly correlated state will now serve as the initial state for magneto-association, providing a much higher overlap to the desired final molecular states. This scheme is similar to the one used for ${ }^{85} \mathrm{Rb}$ in Ref. [39] which not only provided association of dimers but also Efimov trimers [40]. We show that this scheme, which we defined as quenched magneto-association (qMA), substantially improves the efficiency of molecule formation in the ultralow density regime, while still allowing it to be performed within time scales much shorter than those associated with atomic and molecular losses. Our proposed qMA is also found to be superior to tMA at higher densities, thus being of interest for ground-based experiments. More generally, we conjecture that qMA can also be potentially useful for molecular association studies near narrow Feshbach resonances since it provides a scheme to produce a strongly correlated state for associa- 
tion regardless of the strength of the resonance. Keeping in mind the relevant case of ${ }^{87} \mathrm{Rb}^{41} \mathrm{~K}$ mixtures available at CAL, our manuscript is organized as follows. In Section [I] we describe our theoretical model and emphasize its main assumptions and approximations. Section III details both tMA and qMA schemes and analyzes the important time scales associated with the atomic and molecular losses. In Section IV we present our results for association of ${ }^{87} \mathrm{Rb}^{41} \mathrm{~K}$ Feshbach molecules and discuss the main advantages of qMA while verifying its fundamental differences to tMA schemes.

\section{THEORETICAL MODEL}

In our present study we adopted two major assumptions that allow for a qualitative description of magnetoassociation while still providing a clear physical picture on how medium (density) affects the association process. For the interatomic interactions we assume a single channel interaction model between ${ }^{87} \mathrm{Rb}$ and ${ }^{41} \mathrm{~K}$ atoms, given by a Lennard-Jones potential

$$
v(r)=-\frac{C_{6}}{r^{6}}\left(1-\frac{\lambda^{6}}{r^{6}}\right)
$$

where $C_{6} \approx 4274 \mathrm{a}_{0}^{6} E_{\mathrm{h}}[41]$ is the van der Waals' dispersion coefficient and $\lambda$ is a tunable parameter adjusted to provide the desired value of the scattering length. A more realistic description of the interactions between alkali atoms, however, is multichannel in nature and includes the hyperfine interactions responsible for the $B$ field dependence of the scattering length used in experiments with Feshbach resonances. A single channel description of this phenomena is supported by the universal properties of the system [32] whenever $|a| \gg r_{\mathrm{vdW}}$, where $r_{\mathrm{vdW}}=\left(2 \mu C_{6} / \hbar^{2}\right)^{1 / 4} / 2$ is the van de Waals length and $\mu$ the two-body reduced mass. In our present study we model the ${ }^{87} \mathrm{Rb}^{4}{ }^{41} \mathrm{~K}$ interactions near the well-known Feshbach resonance for atoms in the $\left|f=1, m_{f}=1\right\rangle$ hyperfine state (see Fig. 1) by adjusting the values of $\lambda$ in Eq. (11) for each value of $B$ to produce the same value of $a$. As usual, for $B$-fields near a Feshbach resonance the scattering length is well represented by

$$
a(B)=a_{\mathrm{bg}}\left(1-\frac{\Delta B}{B-B_{0}}\right),
$$

where the position of the resonance is $B_{0} \approx 39.4 \mathrm{G}$, its width $\Delta B \approx 37 \mathrm{G}$, and background scattering length $a_{\mathrm{bg}} \approx 284 \mathrm{a}_{0}[42]$.

In order to incorporate density effects to properly describe magneto-association in ultracold quantum gases, we have employed the local density model [43 48], allowing for a physically meaningful way to qualitatively describe the density dependence of various few-body observables. This model introduces a harmonic confinement to the few-body Hamiltonian, whose strength is adjusted to produce a few-body "density" that matches that of the

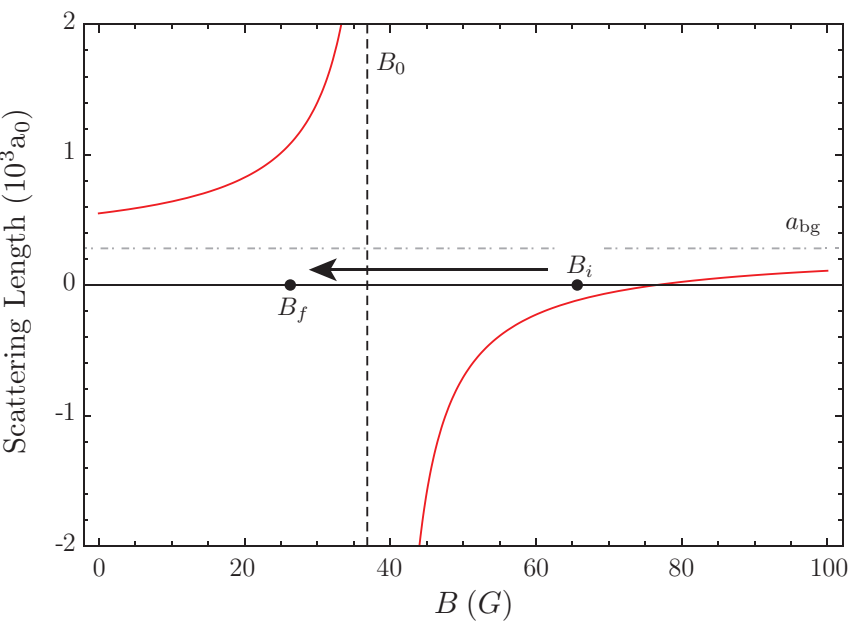

FIG. 1. Scattering length (in units of the Bohr radius, $a_{0}$ ) as a function of applied $B$-field near the ${ }^{87} \mathrm{Rb}-{ }^{41} \mathrm{~K}$ Feshbach resonance at $B_{0} \approx 39.4 \mathrm{G}$. This resonance is characterized by a width $\Delta B \approx 37 \mathrm{G}$ and background scattering length $a_{\mathrm{bg}} \approx 284 \mathrm{a}_{0}[42]$. Both ${ }^{87} \mathrm{Rb}$ and ${ }^{41} \mathrm{~K}$ atoms are in the $\left|f=1, m_{f}=1\right\rangle$ hyperfine state. $B_{i}$ and $B_{f}$ indicate, respectively, the initial and final values of the $B$-field in a hypothetical magneto-association scheme.

experiment. In our current study, the two-atom Hamiltonian is then written as

$$
\hat{H}=-\frac{\hbar^{2}}{2 \mu} \nabla^{2}+\frac{\hbar^{2}}{8 \mu a_{h o}^{4}} r^{2}+v(r)
$$

where $a_{\mathrm{ho}}$ is the harmonic oscillator length. We assume that the number densities of $\mathrm{Rb}$ and $\mathrm{K}$ are equal, i.e., $n_{\mathrm{Rb}}=n_{\mathrm{K}}=n$, and relate the oscillator length to the number density as [46]

$$
a_{\mathrm{ho}}=\sqrt{\frac{\pi}{8}}\left(\frac{4 \pi n}{3}\right)^{-1 / 3} .
$$

This relation allow us to connect our few-body analysis with the relevant energy, length, and time scales to the macroscopic system characterized, respectively,

$$
E_{n}=\frac{\left(6 \pi^{2} n\right)^{2 / 3}}{2 \mu} \hbar^{2}, \quad k_{n}=\frac{\sqrt{2 \mu E_{n}}}{\hbar}, \text { and } t_{n}=\frac{\hbar}{E_{n}} .
$$

We note that our model does not take into account quantum degeneracy and phase-space density effects for association of Feshbach resonances as those experimentally observed in Refs. [49 51]. Nevertheless, the qualitative aspects of our analysis (in particular the comparison between tMA and qMA protocols) still persists and should be observed in more elaborate models in which such collective effects are properly accounted for.

As a result, within our model magneto-association can be easily visualized through the two-atom energy spectrum as shown in Fig. 2. The horizontal energy levels in the $1 /\left|k_{n} a\right| \gg 1$ regime correspond to harmonic states 


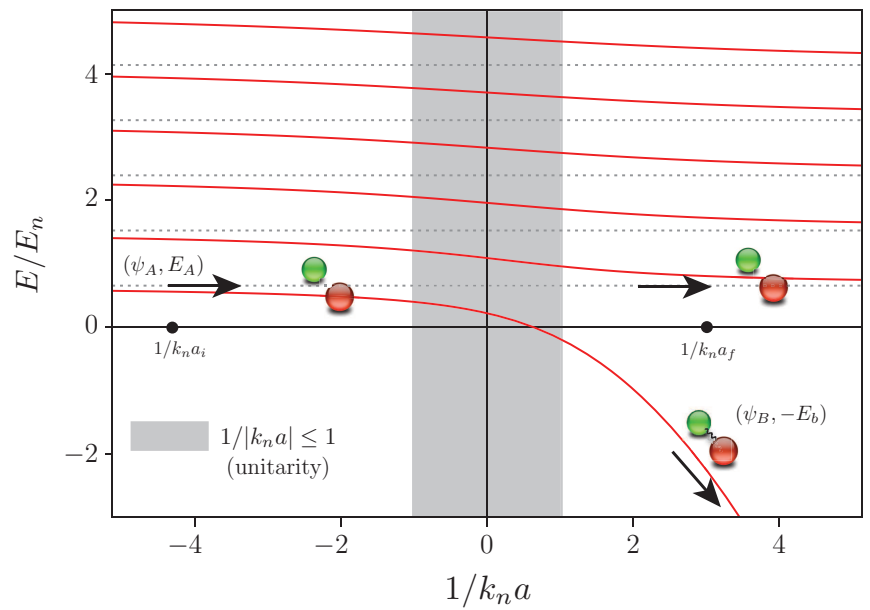

FIG. 2. The energy levels of two atoms in a harmonic trap parameterized by $1 / k_{n} a$ and orbital angular momentum $l=0$ (see text). In the limit of large $1 /\left|k_{n} a\right|$, the spectrum consists of pure harmonic oscillator levels, whose energies are plotted as dashed lines. Positive energies correspond to atomic states, and negative energies represent molecular states. Note that the expected Feshbach molecular state for large positive $a$, having binding energy $E_{b}=\hbar^{2} / 2 \mu a^{2}$, is shifted slightly due to the oscillator potential. The gray region indicate the values in which the system is found in the unitary regime, $1 /\left|k_{n} a\right|<1$.

and represent non-interacting atoms states. The desired target Feshbach molecular state is that with energy given by $-E_{b}=-\hbar^{2} / 2 \mu a^{2}$ for $a>0$ and is also indicated in Fig. 2. [The energy spectrum is obtained by solving the two-body Schrödinger equation for the Hamiltonian in Eq. (3) for the lowest angular momentum state, $l=0$.] The effect of sweeping the $B$-field from high to low (from $B_{i}$ to $B_{f}$ in Fig. (1) corresponds to sweeping $1 / k_{n} a$ from left to right (from $1 / k a_{i}$ to $1 / k_{n} a_{f}$ ) in Fig. 2. Transitions from atomic to molecular states are stronger in the interaction region $1 /\left|k_{n} a\right| \leq 1$, i.e., when interactions are unitary, $n|a|^{3} \geq 1$. Therefore, within our physical picture, magneto-association reduces to the problem of nonadiabatic crossing of energy levels (Landau-Zener) [52]. In our case, multiple levels can participate in the process. Nevertheless, the Landau-Zener (two-level) model still provides a qualitative interpretation of the phenomena and can serve as a guide for understanding of the important parameters controlling molecular association. For instance, within the Landau-Zener model [52] the probability of transitioning from an energy level $\epsilon_{1}$ to $\epsilon_{2}$ via a linear sweep, $\epsilon_{1}-\epsilon_{2}=\alpha_{\epsilon} t$, where $\alpha_{\epsilon}$ is the sweeping rate, is given by

$$
P_{L Z}=1-e^{-2 \pi \Gamma} .
$$

Here, $\Gamma=\epsilon_{12}^{2} / \hbar \alpha_{\epsilon}$ with $\epsilon_{12}$ being the coupling between states $\epsilon_{1}$ and $\epsilon_{2}$. Applying this picture to our case in Fig. 2, where energies are given in units of $E_{n}$ (5), allows us to access important information. For instance, since efficient association is obtained for $\Gamma \gg 1$, the sweeping rates are required to be $\alpha_{\varepsilon} \propto n^{4 / 3}$, which can be too slow in the ultralow density regime of CAL. We will explore these issues next and provide an alternative approach to circumvent this limitation.

\section{QUENCHED MAGNETO-ASSOCIATION}

As already anticipated from the discussion on the previous section, tMA schemes, i.e., applying a linear $B$-field sweep across a Feshbach resonance, might be inefficient for the low density regime relevant to a microgravity environment. Here we detail tMA and we discuss an alternative scheme that overcomes its limitations, but that can also be applied to ground based-experiments. We also discuss and characterize atomic and molecular losses.

\section{A. Sweeps and Quenches}

Within our model, the key physical aspect that makes tMA inefficient at low densities is the fact that during the $B$-field sweep the system remains in the interaction region, $1 /\left|k_{n} a\right| \leq 1$, for a too short amount of time, thus requiring slow sweeps. The tMA scheme is illustrated in Fig. 3(a), where the $B$-field is linearly swept from $B_{i}$ to $B_{f}$ during a time $t_{\mathrm{sw}}$. In order to determine the interaction time, $t_{\mathrm{u}}$, we assume $B(t)=B_{i}-\alpha_{B} t$, where $\alpha_{B}=\left|B_{f}-B_{i}\right| / t_{\mathrm{sw}} \approx 26.03 \mathrm{G} / t_{\mathrm{sw}}$ is the sweep rate, and determine values of $B$ from Eq. (2) in which the condition $1 /\left|k_{n} a\right| \leq 1$ is satisfied. After some algebra one arrives at the interaction time given by

$$
t_{\mathrm{u}} \approx 2\left|\frac{\left(k_{n} a_{\mathrm{bg}}\right) \Delta}{\alpha_{B}}\right| \propto n^{1 / 3} t_{\mathrm{sw}}
$$

As a result, during a given sweep, atoms in the relevant states interact only during a much reduced amount of time as $n \rightarrow 0$.

In order to improve on the interaction time, and consequently the association efficiency, we propose the scheme illustrated in Fig. 3(b). In such a scheme, the system is first quenched to $1 /\left|k_{n} a\right|=0$, corresponding to changing the $B$-field from $B_{i}$ to $B_{0}$ within time scales much shorter than $t_{n}$. We note that at low densities the technical aspects of quenching the $B$-field becomes increasingly easier since $t_{n}$ increases as $n$ decreases. For our studies we assume that the quench is performed instantaneously. After the quench, we allow the system to dwell for a time $t_{\mathrm{dw}}$ at $1 /\left|k_{n} a\right|=0$, thus letting interactions evolve before finally sweeping the field to its final value, $B_{f}$, accordingly to $B(t)=B_{0}-\alpha_{B}^{*} t$, where $\alpha_{B}^{*}=\left|B_{f}-B_{0}\right| / t_{\mathrm{sw}} \approx 1.51 \mathrm{G} / t_{\mathrm{sw}}$. As a result, in quenched magneto-association (qMA) the interaction time is now given by

$$
t_{\mathrm{u}}^{*}=t_{\mathrm{dw}}+\frac{t_{\mathrm{u}}}{2}=t_{\mathrm{dw}}+\underbrace{\left|\frac{\left(k_{n} a_{\mathrm{bg}}\right) \Delta}{\alpha_{B}^{*}}\right|}_{\propto n^{1 / 3} t_{\mathrm{sw}}},
$$


(a) Linear sweep

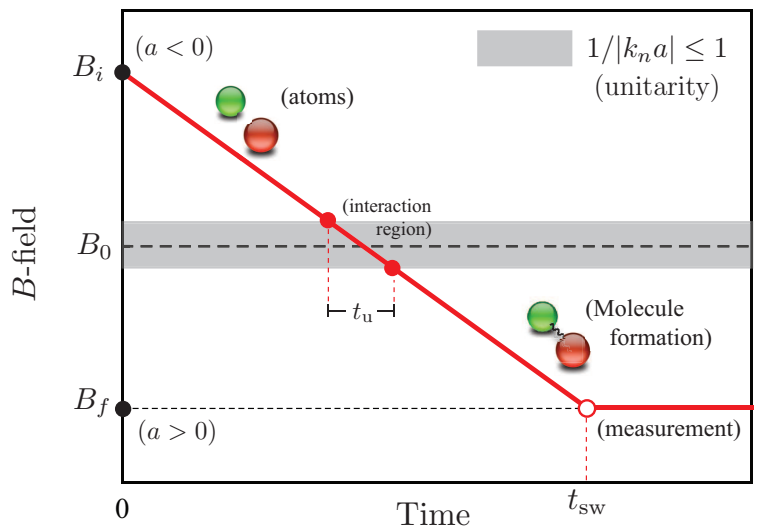

(b) Quench+sweep

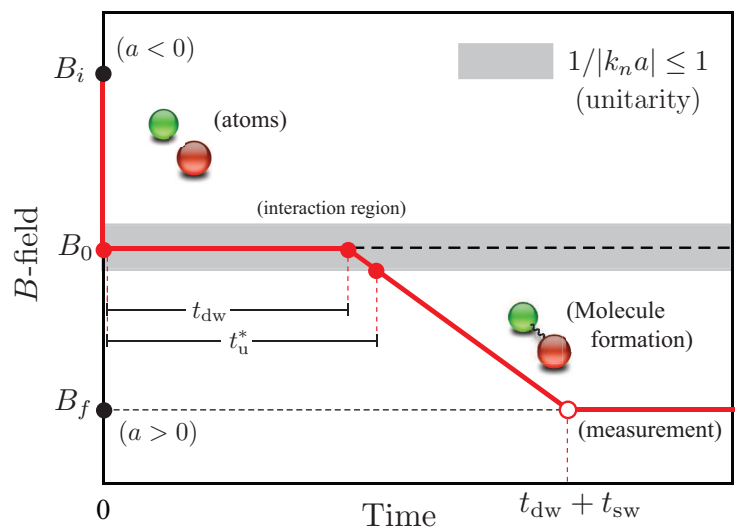

FIG. 3. Schematic of magnetic field vs time in the traditional and quenched magneto-association schemes. (a) In traditional magneto-association (tMA) $B$-field swept from $B_{i}$ to $B_{f}$ at a constant rate during a time $t_{\mathrm{sw}}$. (b) In quenched magnetoassociation (qMA) the $B$-field is instantaneously quenched from $B_{i}$ to $B_{0}$, remaining at $1 /\left|k_{n} a\right|=0$ for a dwell time, $t_{\mathrm{dw}}$, followed by a linear sweep from $B_{0}$ to $B_{f}$. This schematic figure is not to scale, and $B_{i}$ and $B_{f}$ will not, in general, be equidistant from $B_{0}$, nor will $t_{\mathrm{sw}}$ and $t_{\mathrm{dw}}$ bear any particular relationship to each other. In the figure, $t_{\mathrm{u}}$ and $t_{\mathrm{u}}^{*}$ represent the interaction time, i.e., the time in which experience $1 /\left|k_{n} a\right|<1$, for tMA and qMA, respectively.

which can be substantially enhanced by controlling $t_{\mathrm{dw}}$. We note that this scheme is also similar to the one explored in Ref. 31], though assuming $t_{\mathrm{dw}} \approx 0$, and obtaining an efficiency of about $30 \%$ at densities of $10^{12} / \mathrm{cm}^{3}$.

Numerically, we study both tMA and qMA using the time propagation methodology developed in Refs. [40, 53., with a few caveats introduced by the quench. For tMA the initial state for propagation is a pure state, i.e., it is given by

$$
\Psi_{i} \equiv \psi_{A}^{a=a_{i}}(\mathbf{r})
$$

where $\psi_{A}$ is an eigenstate of energy $E_{A}$ for $a=a_{i}$ (see Fig. 2). In the quenched case, however, the initial state for propagation is instead a superposition of states given by

$$
\Psi_{i} \equiv \sum_{\beta} c_{\beta} \exp \left[-\frac{i E_{\beta} t_{\mathrm{dw}}}{\hbar}\right] \psi_{\beta}^{a= \pm \infty}(\mathbf{r})
$$

where $c_{\beta}=\left\langle\psi_{A} \mid \psi_{\beta}\right\rangle$, with $\psi_{\beta}$ and $E_{\beta}$ being the eigenstates and energies of the system at $a= \pm \infty$. As we will see in Section IV the dependence on $t_{\mathrm{dw}}$ in this state plays a crucial role that improves the efficiency of magneto-association by letting interactions evolve at $1 / k_{n} a=0$. Note also that for $t_{\mathrm{dw}} \gg t_{n}$ we expect that truly many-body effects to take place, potentially playing a rule in qMA. Current models do not not capture this physics properly, so we will keep our study within modest values of $t_{\mathrm{dw}}$. Before we compare in details both tMA and qMA schemes of Fig. 3. we must first analyse the stability of the system with respect to losses, as done in the next section.

\section{B. Atomic and molecular losses}

Regardless of the particular magneto-association scheme adopted, few-body losses can drastically reduce the efficiency of molecule formation. Although such loss processes are in general well understood in ultracold atomic and molecular gases 54], magneto-association is a dynamical process and the full understanding on how losses occur as the interactions evolve is nontrivial. In this section, however, we present an analysis that offers a qualitative understanding of the major loss processes, thus helping us to characterize and identify experimental regimes that are likely to mitigate their harmful effects.

In magneto-association the two major few-body loss processes are three-body recombination, the process in which three free atoms collide to produce an atom and diatomic molecule, and atom-molecule vibrational relaxation, causing a de-excitation of the molecular state. Both processes release enough kinetic energy to make their products to escape from typical traps [54]. In order to gain some insight on the time scales of the loss rates and their dependence on the experimentally relevant parameters, we will consider the loss rates only in the regime at which they are maximal, i.e., $1 /\left|k_{n} a\right|<1$, which is the regime most relevant to magneto-association. This analysis should provide an upper limit for the lifetime of the sample during the magneto-association process.

It is well known that three-body recombination rate in the regime $1 /\left|k_{n} a\right|<1$ becomes independent of the scattering length and estimated as 54

$$
L_{3}^{\mathrm{u}}(T)=\frac{4 \pi^{2} \hbar^{5}}{\mu_{3 \mathrm{~b}}^{3}\left(k_{B} T\right)^{2}}\left(1-e^{-4 \eta}\right),
$$

where $\mu_{3 \mathrm{~b}}^{2}=m_{1} m_{2} m_{3} /\left(m_{1}+m_{2}+m_{3}\right)$ is the three-body reduced mass, $T$ is the temperature, and $\eta$ is the threebody inelasticity parameter, which provides a measure 

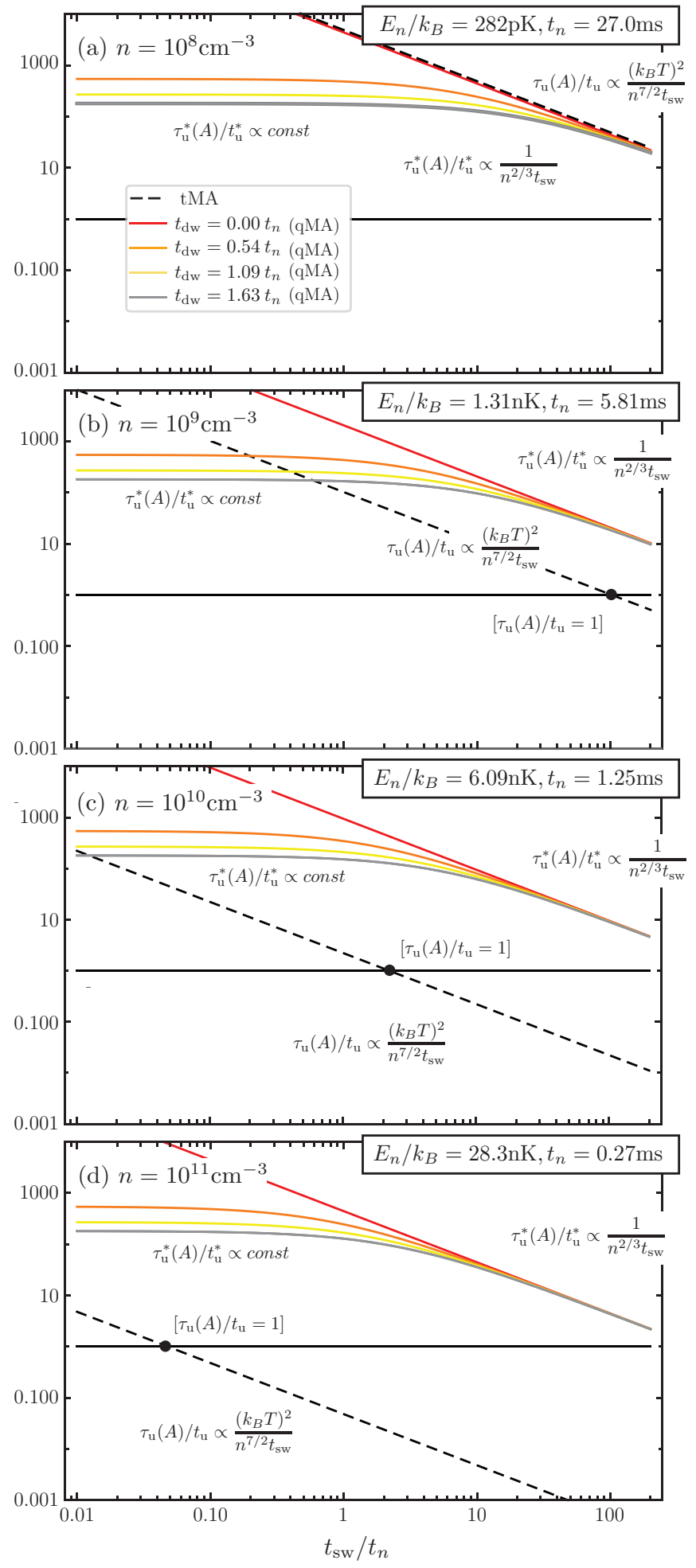

FIG. 4. Analysis of the relevant time scales for magnetoassociation. For tMA, the values for $\tau_{\mathrm{u}} / t_{\mathrm{u}}$ calculated from the lifetimes due to RbRbK losses at $T=100 \mathrm{pK}$ (black-dashed curves) for various densities and a broad range of sweeping times. As $n$ increases, the values of $t_{\mathrm{sw}}$ in which $\tau_{\mathrm{u}} / t_{\mathrm{u}}>1$ becomes quickly more restrictive. For qMA we display the values for $\tau_{\mathrm{u}}^{*} / t_{\mathrm{u}}^{*}$ (solid curves) for various values of $t_{\mathrm{dw}}$. Regardless of the density, a broad range of $t_{\mathrm{sw}}$ satisfy the favorable condition for magneto-association, $\tau_{\mathrm{u}}^{*} / t_{\mathrm{u}}^{*}>1$. of the probability for inelastic transitions. The parameter $\eta$ is dependent upon the details of the interactions and in general obtained experimentally. For the Rb-K mixture we are interested here, Ref. [55] has determined $\eta \approx 0.12$ for collisions involving two $\mathrm{Rb}$ atoms and one $\mathrm{K}(\mathrm{RbRbK})$, and $\eta \approx 0.02$ for collisions involving two $\mathrm{K}$ atoms and one $\mathrm{Rb}(\mathrm{KKRb})$. Now, assuming that the $B$ field sweep in tMA is performed at constant temperature, the atomic lifetime is given by

$$
\begin{aligned}
\tau_{\mathrm{u}}(A) & =\frac{1}{n^{2} L_{3}^{\mathrm{u}}(T)} \\
& =\frac{9 \pi^{2}\left(k_{B} T\right)^{2} \mu_{3 \mathrm{~b}}^{3}}{8\left(1-e^{-4 \eta}\right) \mu^{3} \hbar^{2}} t_{n}^{3} \propto \frac{\left(k_{B} T\right)^{2}}{n^{2}},
\end{aligned}
$$

thus becoming shorter as the density increases and/or the temperature decreases. As a practical example, the lifetimes due to RbRbK losses at $T=100 \mathrm{pK}$ and densities of $10^{8} / \mathrm{cm}^{3}, 10^{9} / \mathrm{cm}^{3}, 10^{10} / \mathrm{cm}^{3}$, and $10^{11} / \mathrm{cm}^{3}$, are, respectively, $37 t_{n}, 1.7 t_{n}, 0.08 t_{n}$ and $0.0037 t_{n}$, or, equivalently, $1 \mathrm{~s}, 10 \mathrm{~ms}, 0.1 \mathrm{~ms}$ and $1 \mu \mathrm{s}$. In order to qualitatively understand what these lifetimes mean, the time scale of losses needs to be compared to that of the interaction time [Eq. (7)],

$$
\tau_{\mathrm{u}}(A) / t_{\mathrm{u}} \propto \frac{\left(k_{B} T\right)^{2}}{n^{7 / 3} t_{\mathrm{sw}}} .
$$

This indicates that an increase in the density, or a decrease in temperature, must be accompanied by a decrease of the sweeping time $t_{\mathrm{sw}}$ or, equivalently, an increase of the sweep rate $\alpha_{B}$, in order to compensate for the increase of atomic losses. As a result, since faster $B$-field sweeps reduce efficiency, obtain a good balance of losses and interction time, $\tau_{\mathrm{u}} / t_{\mathrm{u}}>1$, can only be done at the risk of compromising efficiency. This makes evident that finding the best regime for tMA is dependent upon a balance of various factors. Figure 4 shows the values for $\tau_{\mathrm{u}} / t_{\mathrm{u}}$ calculated from the lifetimes due to RbRbK losses at $T=100 \mathrm{pK}$ (black-dashed curves) for various densities and a broad range of sweeping times. Note that as $n$ increases, the values of $t_{\mathrm{sw}}$ in which $\tau_{\mathrm{u}} / t_{\mathrm{u}}>1$ becomes quickly more restrictive. For each density, we have indicated the corresponding values for $E_{n}$ and $t_{n}$ in relevant units.

In the case of qMA the key difference that improves the time scales for losses is that the quench itself increases the gas temperature and, according to Eq. (11), reduces the loss rates. Assuming that initial temperature is smaller than $E_{n}$, the quench sets the temperature to $k_{B} T=E_{n}$ [56], thus leading to an atomic lifetime determined by

$$
\begin{aligned}
\tau_{\mathrm{u}}^{*}(A) & =\frac{1}{n^{2} L_{3}^{\mathrm{u}}\left(E_{n} / k_{B}\right)} \\
& =\frac{9 \pi^{2} \mu_{3 \mathrm{~b}}^{3}}{8\left(1-e^{-4 \eta}\right) \mu^{3}} t_{n} \propto \frac{1}{n^{2 / 3}} .
\end{aligned}
$$

Interestingly, the lifetime now is linearly proportional to $t_{n}$ and consequently automatically rescaling for different densities and providing a weaker dependence on 
density than Eq. (12). For instance, now the lifetimes due to RbRbK losses at $T=100 \mathrm{pK}$ is $295 t_{n}$, which for densities of $10^{8} / \mathrm{cm}^{3}, 10^{9} / \mathrm{cm}^{3}, 10^{10} / \mathrm{cm}^{3}$, and $10^{11} / \mathrm{cm}^{3}$, become $8 \mathrm{~s}, 1.7 \mathrm{~s}, 370 \mathrm{~ms}$, and $80 \mathrm{~ms}$, respectively. Comparing this lifetime to the interaction time in Eq. (8), we obtain in the limit of long dwell times, $t_{\mathrm{dw}} \gg t_{\mathrm{u}}$, an interaction time of $t_{\mathrm{u}}^{*} \approx t_{\mathrm{dw}}\left(\propto t_{n}\right)$, and

$$
\tau_{\mathrm{u}}^{*}(A) / t_{\mathrm{u}}^{*} \propto \text { const. }
$$

In the other hand, limit of short dwell times, $t_{\mathrm{dw}} \ll t_{\mathrm{u}}$ the interaction time is $t_{\mathrm{u}}^{*} \approx t_{\mathrm{u}} / 2\left(\propto t_{n}\right)$, and

$$
\tau_{\mathrm{u}}^{*}(A) / t_{\mathrm{u}}^{*} \propto \frac{1}{n^{2 / 3} t_{\mathrm{sw}}} .
$$

In either case, the above analysis indicates that qMA provides a substantially more favorable regime with respect to the scaling of losses with density and sweeping times. Figure 4 demonstrate this by displaying the values for $\tau_{\mathrm{u}}^{*} / t_{\mathrm{u}}^{*}$, also calculated from the lifetimes due to RbRbK (solid curves), for various values of $t_{\mathrm{dw}}$. Note that regardless of the density, a broad range of $t_{\mathrm{sw}}$ satisfy the favorable condition for association, $\tau_{\mathrm{u}}^{*} / t_{\mathrm{u}}^{*}>1$, a result much superior from those from tMA.

A similar analysis of the lifetime can be also provided once molecular association take places and the relevant loss process is atom-molecule relaxation. Here, too, loss rates in the regime $1 /\left|k_{n} a\right|<1$ become independent on the scattering length and estimated as 54

$$
\beta_{\mathrm{u}}(T)=\frac{2^{1 / 2} \pi^{1 / 2} \hbar}{\mu_{\mathrm{ad}}^{3 / 2}\left(k_{B} T\right)^{1 / 2}}\left(1-e^{-4 \eta}\right),
$$

where $\mu_{\mathrm{ad}}=\left(m_{1}+m_{2}\right) m_{3} /\left(m_{1}+m_{2}+m_{3}\right)$ is the atommolecule reduced mass. In this case, assuming that the $B$-field sweep in tMA is performed at constant temperature, the molecular lifetime is simply given by

$$
\begin{aligned}
\tau_{\mathrm{u}}(M) & =\frac{1}{n \beta_{\mathrm{u}}(T)} \\
& =\frac{3 \pi^{3 / 2} \mu_{\mathrm{ad}}^{3 / 2}\left(k_{B} T\right)^{1 / 2} \hbar^{1 / 2}}{2 \mu^{3 / 2}\left(1-e^{-4 \eta}\right)} t_{n}^{3 / 2} \propto \frac{\left(k_{B} T\right)^{1 / 2}}{n},
\end{aligned}
$$

providing a weaker dependence on temperature and density than those for their atomic counterparts in Eq. (12). From the above equation, the molecular lifetimes due to RbRbK losses at $T=100 \mathrm{pK}$ and densities of $10^{8} / \mathrm{cm}^{3}$, $10^{9} / \mathrm{cm}^{3}, 10^{10} / \mathrm{cm}^{3}$, and $10^{11} / \mathrm{cm}^{3}$, are, respectively, $29 t_{n}, 14 t_{n}, 6.3 t_{n}$ and $2.9 t_{n}$, or, equivalently, $0.8 \mathrm{~s}, 80 \mathrm{~ms}$, $8 \mathrm{~ms}$ and $0.8 \mathrm{~ms}$. These lifetimes are to be compared to that of the interaction time [Eq. (7)],

$$
\tau_{\mathrm{u}}(M) / t_{\mathrm{u}} \propto \frac{\left(k_{B} T\right)^{1 / 2}}{n^{4 / 3} t_{\mathrm{sw}}} .
$$

In the case of qMA, assuming that initial temperature is smaller than $E_{n}$, the molecular lifetime determined by

$$
\begin{aligned}
\tau_{\mathrm{u}}^{*}(M) & =\frac{1}{n \beta_{\mathrm{u}}\left(E_{n} / k_{B}\right)} \\
& =\frac{3 \pi^{3 / 2} \mu_{\mathrm{ad}}^{3 / 2} \hbar}{2 \mu^{3 / 2}\left(1-e^{-4 \eta}\right)} t_{n} \propto \frac{1}{n^{2 / 3}},
\end{aligned}
$$

Here, the lifetimes due to RbRbK losses at $T=100 \mathrm{pK}$ is $49 t_{n}$, which for densities of $10^{8} / \mathrm{cm}^{3}, 10^{9} / \mathrm{cm}^{3}, 10^{10} / \mathrm{cm}^{3}$, and $10^{11} / \mathrm{cm}^{3}$, become $1.3 \mathrm{~s}, 0.3 \mathrm{~s}, 62 \mathrm{~ms}$, and $13 \mathrm{~ms}$, respectively. Comparing this lifetime to the interaction time in Eq. (8), we obtain in the limit of long dwell times, $t_{\mathrm{dw}} \gg t_{\mathrm{u}}$

$$
\tau_{\mathrm{u}}^{*}(M) / t_{\mathrm{u}}^{*} \propto \text { const },
$$

while for short dwell times, $t_{\mathrm{dw}} \ll t_{\mathrm{u}}$

$$
\tau_{\mathrm{u}}^{*}(M) / t_{\mathrm{u}}^{*} \propto \frac{1}{n^{2 / 3} t_{\mathrm{sw}}} .
$$

The results above, lead to similar conclusions reached when analysing the atomic lifetimes. Note that for the particular case of RbK mixtures, the typical molecular lifetimes $\tau_{M}^{\mathrm{u}}$ and $\tau_{M}^{\mathrm{u} *}$ are longer than those for the atomic lifetimes analysed above.

Overall, the analysis above indicates that the detrimental effects atomic on molecular losses to magnetoassociation are in general mitigated in the low-density regime, regardless of the magneto-association scheme. The CAL fully takes advantage of this property due to its unique capability to provide ultralow-density samples. The qMA, however, seems to provide a more stable scheme, in particular at higher densities, due to its independence of temperature and the much more favorable ratio between lifetime and interaction times, $\tau_{A}^{\mathrm{u} *} / t_{\mathrm{u}}^{*}$. The caveat of using quenches is that the final temperature of the molecular sample will directly depend on the density (via its dependence on $E_{n}$ ), an effect that can once again mitigated at low-densities. Although such prospects are positive with respect to losses at the low-density regime, the question we now focus is how the actual efficiency of both magneto-association schemes compares to each other in this regime and whether association occurs in time scales shorter than the atomic and molecular losses. We provide such an analysis in the next section.

\section{MOLECULAR ASSOCIATION}

In this section we present our numerical simulation for both tMA and qMA near the ${ }^{87} \mathrm{Rb}^{41} \mathrm{~K}$ Feshbach resonance at $B_{0}=39.4 \mathrm{G}$ [42] (Fig. 10. In each case, we select $a_{i}=-2 r_{\mathrm{vdW}}\left(\approx-144 \mathrm{a}_{0}\right)$ and $a_{f}=100 r_{\mathrm{vdW}}\left(\approx 7230 \mathrm{a}_{0}\right)$, corresponding to $B_{i}=63.92 \mathrm{G}$ and $B_{f}=37.89 \mathrm{G}$. Therefore, we are assuming bosonic heteronuclear Feshbach molecules which are substantially larger than previously studied [57, 58] which aligns best with CAL's future experiments in microgravity 24]. As a result, most of our 

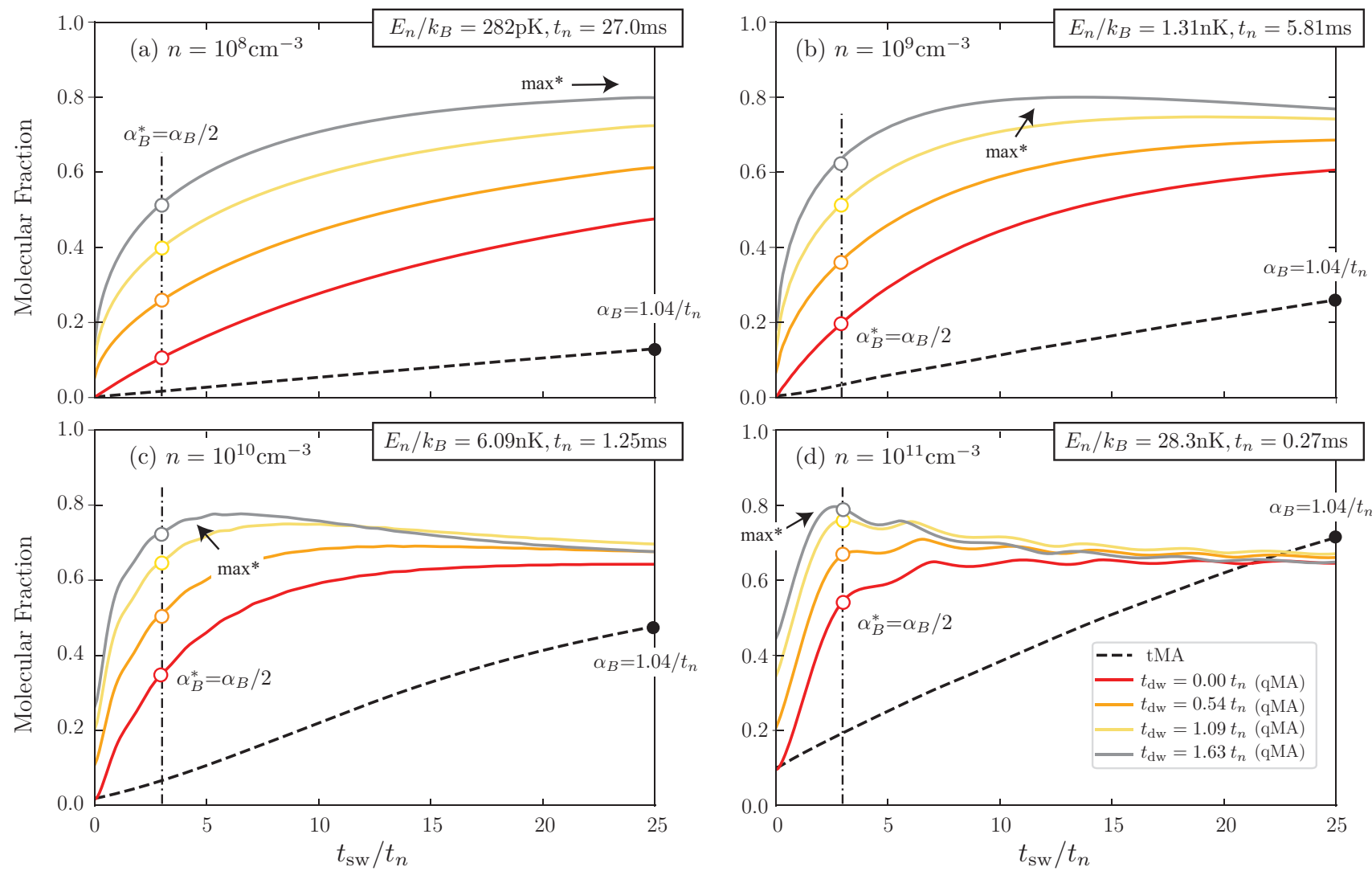

FIG. 5. Fraction of molecules produced as a function of $t_{\mathrm{sw}}$ for various densities. The dashed curve represent results for tMA while solid curves are those for qMA at different dweeling times, $t_{\mathrm{dw}}$. In all cases the initial and final states are characterized by $a_{i}=-2 r_{\mathrm{vdW}}$ and $a_{f}=100 r_{\mathrm{vdw}}$, respectively. Note that at $t_{\mathrm{sw}}=0$, tMA results are identical to those from qMA at $t_{\mathrm{sw}}=t_{\mathrm{dw}}=0$. (See text for more discussions on these results and comparisons.) Figure display the corresponding values for $E_{n}$ and $t_{n}$.

studies presented here will be performed for atomic densities in which the average interatomic distances are larger than the molecular size, i.e., $n \leq 10^{11} / \mathrm{cm}^{3}$. We study the molecular fraction in terms of the sweeping time, $t_{\mathrm{sw}}$, dwell time, $t_{\mathrm{dw}}$, and atomic densities. We note that the qualitative trends in our results do not depend on the particular choices of $B_{i}$ and $B_{f}$, so long as they are chosen off resonance, i.e. $1 /\left|k_{n} a\right| \gg 1$.

Here, we chose to compute the molecular fraction for four different atomic densities $n=10^{8} / \mathrm{cm}^{3}, 10^{9} / \mathrm{cm}^{3}$, $10^{10} / \mathrm{cm}^{3}$, and $10^{11} / \mathrm{cm}^{3}$, thereby covering the density regime available the CAL environment. Results are dis-

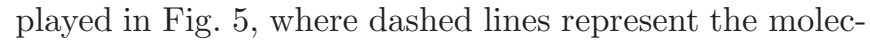
ular fractions obtained via tMA, and solid lines the ones obtained via qMA for different values of $t_{\mathrm{dw}}$ [see colorcoded legend on the inset of Fig. 5(d)]. For each density, we also have indicated the corresponding values for $E_{n}$ and $t_{n}$ as they characterize the typical energies and time scales of the system at a given density $n$. These calculations yield a few crucial observations, valid for all densities considered.

First, as shown in Fig. 5, in the low-density regime qMA produces a higher molecular fraction than tMA within the same value for $t_{\mathrm{sw}}$. This immediately im- plies the most significant result of this study: within a given $t_{\mathrm{sw}}$, a quench with finite $t_{\mathrm{dw}}$ followed by a $B$ field sweep away from unitarity will in general produce a larger molecular fraction than a pure $B$-field sweep in the same amount of time. Therefore, in order to produce a larger fraction of molecules in the shortest amount of time, qMA is clearly the optimal choice. The faster times for molecular formation within qMA is crucially important since it ensures the mitigation of atomic and molecular losses, in particular for higher densities, as discussed in Section. IIIB (see Fig. 4). Figure 5 also indicates that qMA reaches an efficiency of about $80 \%$, reaching this maximum value for smaller values of $t_{\mathrm{sw}} / t_{n}$ values as $n$ increases (see points in Figure 5 marked by "max*"). The finite efficiency in qMA is likely to be associated to the nature of the quenched state in Eq. (10), where excited states are more likely to remain in the atomic states than the target molecular state which the $B$-field is swept away from the interaction region $1 /\left|k_{n} a\right|<1$. A more complete analysis on this topic is beyond the scope of our present study. For tMA, although one would in theory expect a nearly $100 \%$ efficiency as $t_{\mathrm{sw}} \rightarrow \infty$, the results in Fig. [5, along with those for the atomic losses in Fig. 4, clearly shows that substantial losses will take place well 
before being able to reach this level of efficiency.

In order to better understand the physical aspects determining the superiority of qMA over tMA we look at the values of the molecular fraction at $t_{\mathrm{sw}}=0$ in Fig. 5 This value indicates the quality of the overlap between the initial state (prior the $B$-field sweeping) and the final molecular state. As shown in Fig. [5 while for tMA and qMA $\left(t_{\mathrm{dw}}=0\right)$ the molecular fraction are nearly identical, it increases for qMA with $t_{\mathrm{dw}}$. This is consistent with the experimental observations in Ref. 31] and demonstrates that the dwelling time plays an important role in the state preparation by letting correlations evolve in the interaction region $1 /\left|k_{n} a\right|<1$. We note, however, that for $t_{\mathrm{sw}}>0$ the sweeping rates implied in Fig. 罒are, in fact, different. While for the tMA the sweep rate is $\alpha_{B}=\left|B_{f}-B_{i}\right| / t_{\mathrm{sw}} \approx 26.03 \mathrm{G} / t_{\mathrm{sw}}$ in qMA the rate is $\alpha_{B}^{*}=\left|B_{f}-B_{0}\right| / t_{\mathrm{sw}} \approx 1.51 \mathrm{G} / t_{\mathrm{sw}}$. The slower values for $\alpha_{B}^{*}$ thus partially explains the higher molecular fraction obtained via qMA for a given value of $t_{\mathrm{sw}}>0$.

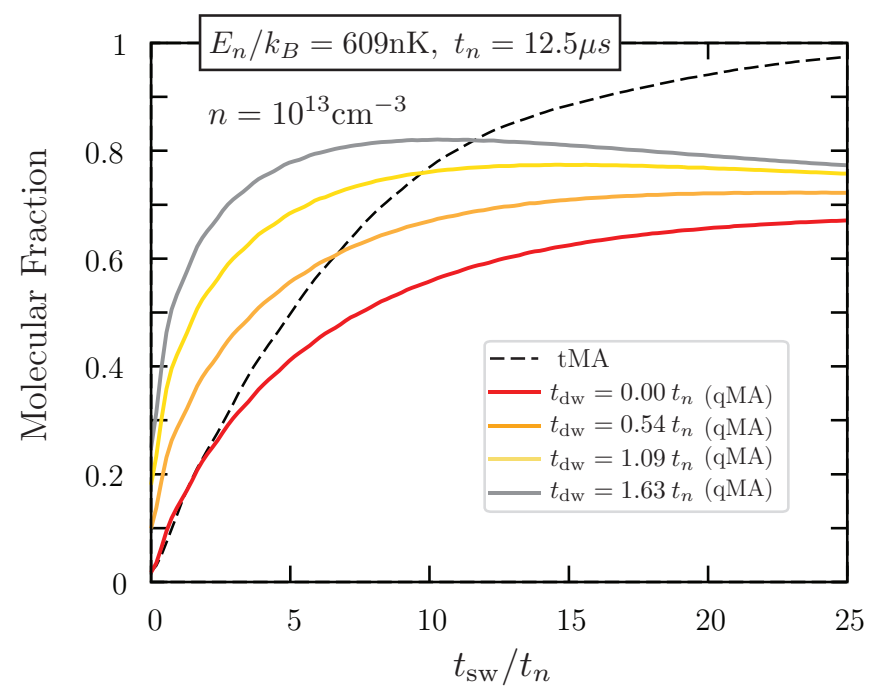

FIG. 6. Fraction of molecules produced as a function of $t_{\mathrm{sw}}$ for $n=10^{13} / \mathrm{cm}^{3}$. The dashed curve represent results for tMA while solid curves are those for qMA at different dweeling times, $t_{\mathrm{dw}}$. In all cases the initial and final states are characterized by $a_{i}=-2 r_{\mathrm{vdW}}$ and $a_{f}=10 r_{\mathrm{vdw}}$, respectively.

In order to make a more direct comparison between the efficiency of both schemes we should compare, for instance, the molecular fractions obtained for sweeping rates that leads to the same sweeping times within the interaction region, $1 /\left|k_{n} a\right|=1$. Now, considering that for tMA one expands twice the time sweeping in this region than for qMA, we will seek for values of $t_{\mathrm{sw}}$ in which $\alpha_{B}^{*}=\alpha_{B} / 2$. We note, however, since in tMA and qMA the system experiences interactions differently during the time spent sweeping across the $1 /\left|k_{n} a\right|<1$, our comparison here can only provides a rough analysis. Our comparison is shown in Fig. 5 We indicate the molecular fraction for tMA at $t_{\mathrm{sw}}=25 t_{n}$ by a solid circle, producing the rate $\alpha_{B}=1.04 / t_{n}$. This value should then be compare to those obtained via qMA at $t_{\mathrm{sw}}=2.90 t_{n}$, as indicated by the open circles in Fig. 5. This analysis again demonstrate that for low densities, as $t_{\mathrm{dw}}$ increases, the efficiency of qMA will surpass that of tMA, while still providing a much faster scheme to associate atoms into molecules.

In order to illustrate the comparative performance of both association schemes at higher densities, and other typical parameters, Figure 6 shows our simulations for tMA and qMA for an atomic density of $n=10^{13} / \mathrm{cm}^{3}$. This calculation associate atoms to a Feshbach molecular state with $a=10 r_{\mathrm{vdW}}$ and demonstrate again the superiority of qMA in creating molecules at a faster rate. This indicates that qMA not only provides a vital tool for molecular creation in microgravity, but also in typical regimes in ground-based experiments.

\section{CONCLUSION}

In this manuscript we have investigated methods to produce molecules via magneto-association near a Feshbach resonance, focusing on the low-density regime relevant to the microgravity environment of CAL. Based on the trends discovered from our computations, we conclude that a qMA can generally be made superior to tMA. Within qMA, we found that the dwelling time at the interaction region, $1 /\left|k_{n} a\right|<1$, allows for correlations to develop, thus providing a much more efficient scheme for association of atoms in molecules. Our results show that qMA allows for a much higher association efficiency $(\sim 80 \%)$ within considerably faster time scales than tMA. This allows for further mitigation of atomic and molecular losses, regardless of the density regime.

In further studies of molecular production, several more complicated aspects of the system could be investigated. For instance, one could introduce dynamically the various loss processes dependent on scattering length, and attempt to optimize molecular production constrained by the loss timescales in a more rigorous way than presented here. Also, while the analysis in this study considered purely two-body interactions, one could extend the analysis by incorporating three-body effects, which would then have to consider both Rb-Rb$\mathrm{K}$ and $\mathrm{Rb}-\mathrm{K}-\mathrm{K}$ interactions, as well as the formation of triatomic Efimov states existing in the system [39].

\section{ACKNOWLEDGMENTS}

This research was carried out under a contract with the National Aeronautics and Space Administration. KW acknowledges support provided by the Undergraduate Research Opportunities Program (UROP) at the University of Colorado Boulder. JRW and JPD were supported by JPL-CALTECH under NASA contract NNH13ZTT002N. JPD also acknowledges partial support from the U. S. National Science Foundation, grant number PHY-2012125. 
[1] K.-A. B. Soderberg, N. Gemelke, and C. Chin, "Ultracold molecules: vehicles to scalable quantum information processing," New Journal of Physics, vol. 11, p. 055022, may 2009.

[2] L. D. Carr, D. DeMille, R. V. Krems, and J. Ye, "Cold and ultracold molecules: science, technology and applications," New Journal of Physics, vol. 11, p. 055049, may 2009.

[3] J. W. Park, Z. Z. Yan, H. Loh, S. A. Will, and M. W. Zwierlein, "Second-scale nuclear spin coherence time of ultracold 23na40k molecules," Science, vol. 357, no. 6349, pp. 372-375, 2017.

[4] K.-K. Ni, T. Rosenband, and D. D. Grimes, "Dipolar exchange quantum logic gate with polar molecules," Chem. Sci., vol. 9, pp. 6830-6838, 2018.

[5] L. Anderegg, L. W. Cheuk, Y. Bao, S. Burchesky, W. Ketterle, K.-K. Ni, and J. M. Doyle, "An optical tweezer array of ultracold molecules," Science, vol. 365, no. 6458 , pp. 1156-1158, 2019.

[6] R. V. Krems, "Cold controlled chemistry," Phys. Chem. Chem. Phys., vol. 10, pp. 4079-4092, 2008.

[7] G. Quéméner and P. S. Julienne, "Ultracold molecules under control!," Chem. Rev., vol. 112, pp. 4949-5011, 2012.

[8] M.-G. Hu, Y. Liu, D. D. Grimes, Y.-W. Lin, A. H. Gheorghe, R. Vexiau, N. Bouloufa-Maafa, O. Dulieu, T. Rosenband, and K.-K. Ni, "Direct observation of bimolecular reactions of ultracold krb molecules," Science, vol. 366, no. 6469, pp. 1111-1115, 2019.

[9] G. Valtolina, K. Matsuda, W. G. Tobias, J.-R. Li, L. De Marco, and J. Ye, "Dipolar evaporation of reactive molecules to below the fermi temperature," Nature, vol. 588, no. 7837, pp. 239-243, 2020.

[10] K.-K. Ni, S. Ospelkaus, M. H. G. de Miranda, A. Pe'er, B. Neyenhuis, J. J. Zirbel, S. Kotochigova, P. S. Julienne, D. S. Jin, and J. Ye, "A high phase-space-density gas of polar molecules," Science, vol. 322, no. 5899, pp. 231235, 2008.

[11] J. G. Danzl, M. J. Mark, E. Haller, M. Gustavsson, R. Hart, J. Aldegunde, J. M. Hutson, and H.-C. Nägerl, "An ultracold high-density sample of rovibronic groundstate molecules in an optical lattice," Nature Physics, vol. 6, no. 4, pp. 265-270, 2010.

[12] S. Ospelkaus, K.-K. Ni, D. Wang, M. H. G. de Miranda, B. Neyenhuis, G. Quéméner, P. S. Julienne, J. L. Bohn, D. S. Jin, and J. Ye, "Quantum-state controlled chemical reactions of ultracold potassium-rubidium molecules," Science, vol. 327, no. 5967, pp. 853-857, 2010.

[13] K. K. Ni, S. Ospelkaus, D. Wang, G. Quéméner, B. Neyenhuis, M. H. G. de Miranda, J. L. Bohn, J. Ye, and D. S. Jin, "Dipolar collisions of polar molecules in the quantum regime," Nature, vol. 464, no. 7293, pp. 13241328, 2010.

[14] Y. Wang, J. P. D'Incao, and C. H. Greene, "Efimov effect for three interacting bosonic dipoles," Phys. Rev. Lett., vol. 106, p. 233201, Jun 2011.

[15] Y. Wang, J. P. D'Incao, and C. H. Greene, "Universal three-body physics for fermionic dipoles," Phys. Rev. Lett., vol. 107, p. 233201, Nov 2011.

[16] C. Chin and V. V. Flambaum, "Enhanced sensitivity to fundamental constants in ultracold atomic and molecu- lar systems near feshbach resonances," Phys. Rev. Lett., vol. 96, p. 230801, Jun 2006.

[17] C. Chin, V. V. Flambaum, and M. G. Kozlov, "Ultracold molecules: new probes on the variation of fundamental constants," New Journal of Physics, vol. 11, p. 055048, may 2009.

[18] A. Borschevsky, K. Beloy, V. V. Flambaum, and P. Schwerdtfeger, "Sensitivity of ultracold-atom scattering experiments to variation of the fine-structure constant," Phys. Rev. A, vol. 83, p. 052706, May 2011.

[19] T. Köhler, K. Góral, and P. S. Julienne, "Production of cold molecules via magnetically tunable feshbach resonances," Rev. Mod. Phys., vol. 78, pp. 1311-1361, Dec 2006.

[20] T. M. Hanna, T. Köhler, and K. Burnett, "Association of molecules using a resonantly modulated magnetic field," Phys. Rev. A, vol. 75, p. 013606, Jan 2007.

[21] T. V. Tscherbul, T. Calarco, I. Lesanovsky, R. V. Krems, A. Dalgarno, and J. Schmiedmayer, "rf-field-induced feshbach resonances," Phys. Rev. A, vol. 81, p. 050701, May 2010.

[22] D. J. Owens, T. Xie, and J. M. Hutson, "Creating feshbach resonances for ultracold molecule formation with radio-frequency fields," Phys. Rev. A, vol. 94, p. 023619, Aug 2016.

[23] Y. Ding, J. P. D'Incao, and C. H. Greene, "Effective control of cold collisions with radio-frequency fields," Phys. Rev. A, vol. 95, p. 022709, Feb 2017.

[24] J. P. D'Incao, M. Krutzik, E. Elliott, and J. R. Williams, "Enhanced association and dissociation of heteronuclear Feshbach molecules in a microgravity environment," Phys. Rev. A, vol. 95, p. 012701, Jan 2017.

[25] P. Giannakeas, L. Khaykovich, J.-M. Rost, and C. H. Greene, "Nonadiabatic molecular association in thermal gases driven by radio-frequency pulses," Phys. Rev. Lett., vol. 123, p. 043204, Jul 2019.

[26] J. Cubizolles, T. Bourdel, S. J. J. M. F. Kokkelmans, G. V. Shlyapnikov, and C. Salomon, "Production of longlived ultracold $\mathrm{li}_{2}$ molecules from a fermi gas," Phys. Rev. Lett., vol. 91, p. 240401, Dec 2003.

[27] J. Herbig, T. Kraemer, M. Mark, T. Weber, C. Chin, H.C. Nägerl, and R. Grimm, "Preparation of a pure molecular quantum gas," Science, vol. 301, no. 5639, pp. 15101513, 2003.

[28] C. A. Regal, C. Ticknor, J. L. Bohn, and D. S. Jin, "Creation of ultracold molecules from a fermi gas of atoms," Nature, vol. 424, no. 6944, pp. 47-50, 2003.

[29] K. E. Strecker, G. B. Partridge, and R. G. Hulet, "Conversion of an atomic fermi gas to a long-lived molecular bose gas," Phys. Rev. Lett., vol. 91, p. 080406, Aug 2003.

[30] S. Dürr, T. Volz, A. Marte, and G. Rempe, "Observation of molecules produced from a bose-einstein condensate," Phys. Rev. Lett., vol. 92, p. 020406, Jan 2004.

[31] M. Mark, T. Kraemer, J. Herbig, C. Chin, H.-C. Nägerl, and R. Grimm, "Efficient creation of molecules from a cesium Bose-Einstein condensate," Europhysics Letters (EPL), vol. 69, pp. 706-712, mar 2005.

[32] C. Chin, R. Grimm, P. Julienne, and E. Tiesinga, "Feshbach resonances in ultracold gases," Rev. Mod. Phys., vol. 82, pp. 1225-1286, Apr 2010. 
[33] K. Bergmann, N. V. Vitanov, and B. W. Shore, "Perspective: Stimulated raman adiabatic passage: The status after 25 years," The Journal of Chemical Physics, vol. 142, no. 17, p. 170901, 2015.

[34] E. R. Elliott, M. C. Krutzik, J. R. Williams, R. J. Thompson, and D. C. Aveline, "Nasa's cold atom lab (cal): system development and ground test status," $n p j$ Microgravity, vol. 4, no. 1, p. 16, 2018.

[35] D. C. Aveline, J. R. Williams, E. R. Elliott, C. Dutenhoffer, J. R. Kellogg, J. M. Kohel, N. E. Lay, K. Oudrhiri, R. F. Shotwell, N. Yu, and R. J. Thompson, "Observation of bose-einstein condensates in an earth-orbiting research lab," Nature, vol. 582, no. 7811, pp. 193-197, 2020.

[36] J. Williams, S. wey Chiow, N. Yu, and H. Müller, "Quantum test of the equivalence principle and space-time aboard the international space station," New Journal of Physics, vol. 18, p. 025018, feb 2016.

[37] P. Asenbaum, C. Overstreet, M. Kim, J. Curti, and M. A. Kasevich, "Atom-interferometric test of the equivalence principle at the $10^{-12}$ level," Phys. Rev. Lett., vol. 125, p. 191101, Nov 2020.

[38] M. S. Safronova, D. Budker, D. DeMille, D. F. J. Kimball, A. Derevianko, and C. W. Clark, "Search for new physics with atoms and molecules," Rev. Mod. Phys., vol. 90, p. 025008, Jun 2018.

[39] C. E. Klauss, X. Xie, C. Lopez-Abadia, J. P. D’Incao, Z. Hadzibabic, D. S. Jin, and E. A. Cornell, "Observation of efimov molecules created from a resonantly interacting bose gas," Phys. Rev. Lett., vol. 119, p. 143401, Oct 2017.

[40] J. P. D'Incao, J. Wang, and V. E. Colussi, "Efimov physics in quenched unitary bose gases," Phys. Rev. Lett., vol. 121, p. 023401, Jul 2018.

[41] A. Derevianko, J. F. Babb, and A. Dalgarno, "Highprecision calculations of van der Waals coefficients for heteronuclear alkali-metal dimers," Phys. Rev. A, vol. 63, p. 052704, Apr 2001.

[42] A. Simoni, M. Zaccanti, C. D'Errico, M. Fattori, G. Roati, M. Inguscio, and G. Modugno, "Near-threshold model for ultracold KRb dimers from interisotope Feshbach spectroscopy," Phys. Rev. A, vol. 77, p. 052705, May 2008.

[43] B. Borca, D. Blume, and C. H. Greene, "A two-atom picture of coherent atom-molecule quantum beats," New Journal of Physics, vol. 5, pp. 111-111, sep 2003.

[44] K. Góral, T. Köhler, S. A. Gardiner, E. Tiesinga, and P. S. Julienne, "Adiabatic association of ultracold molecules via magnetic-field tunable interactions," Journal of Physics B: Atomic, Molecular and Optical Physics, vol. 37, pp. 3457-3500, aug 2004.

[45] J. von Stecher and C. H. Greene, "Spectrum and Dynamics of the BCS-BEC Crossover from a Few-Body Perspec- tive," Phys. Rev. Lett., vol. 99, p. 090402, Aug 2007.

[46] A. G. Sykes, J. P. Corson, J. P. D'Incao, A. P. Koller, C. H. Greene, A. M. Rey, K. R. A. Hazzard, and J. L. Bohn, "Quenching to unitarity: Quantum dynamics in a three-dimensional Bose gas," Phys. Rev. A, vol. 89, p. 021601, Feb 2014.

[47] J. P. Corson and J. L. Bohn, "Bound-state signatures in quenched Bose-Einstein condensates," Phys. Rev. A, vol. 91, p. 013616, Jan 2015.

[48] V. E. Colussi, J. P. Corson, and J. P. D'Incao, "Dynamics of three-body correlations in quenched unitary bose gases," Phys. Rev. Lett., vol. 120, p. 100401, Mar 2018.

[49] E. Hodby, S. T. Thompson, C. A. Regal, M. Greiner, A. C. Wilson, D. S. Jin, E. A. Cornell, and C. E. Wieman, "Production efficiency of ultracold feshbach molecules in bosonic and fermionic systems," Phys. Rev. Lett., vol. 94, p. 120402, Mar 2005.

[50] S. T. Thompson, E. Hodby, and C. E. Wieman, "Ultracold molecule production via a resonant oscillating magnetic field," Phys. Rev. Lett., vol. 95, p. 190404, Nov 2005.

[51] S. B. Papp and C. E. Wieman, "Observation of heteronuclear feshbach molecules from a ${ }^{85} \mathrm{Rb}--{ }^{87} \mathrm{Rb}$ gas," Phys. Rev. Lett., vol. 97, p. 180404, Oct 2006.

[52] C. W. Clark, "The calculation of non-adiabatic transition probabilities," Physics Letters A, vol. 70, no. 4, pp. 295296, 1979.

[53] J. E. Bækhøj, O. I. Tolstikhin, and L. B. Madsen, "Slow' time discretization: a versatile time propagator for the time-dependent Schrödinger equation," Journal of Physics B: Atomic, Molecular and Optical Physics, vol. 47, p. 075007, mar 2014.

[54] J. P. D'Incao, "Few-body physics in resonantly interacting ultracold quantum gases," Journal of Physics B: Atomic, Molecular and Optical Physics, vol. 51, p. 043001, jan 2018.

[55] G. Barontini, C. Weber, F. Rabatti, J. Catani, G. Thalhammer, M. Inguscio, and F. Minardi, "Observation of heteronuclear atomic efimov resonances," Phys. Rev. Lett., vol. 103, p. 043201, Jul 2009.

[56] P. Makotyn, C. E. Klauss, D. L. Goldberger, E. A. Cornell, and D. S. Jin, "Universal dynamics of a degenerate unitary bose gas," Nature Physics, vol. 10, no. 2, pp. 116119, 2014.

[57] C. Klempt, T. Henninger, O. Topic, M. Scherer, L. Kattner, E. Tiemann, W. Ertmer, and J. J. Arlt, "Radio-frequency association of heteronuclear feshbach molecules," Phys. Rev. A, vol. 78, p. 061602, Dec 2008.

[58] C. Weber, G. Barontini, J. Catani, G. Thalhammer, M. Inguscio, and F. Minardi, "Association of ultracold double-species bosonic molecules," Phys. Rev. A, vol. 78, p. 061601, Dec 2008. 